Handgelenk und Handwurzel

\section{Resektion des intakten Diskus bei ulnokarpalem Impaktionssyndrom}

Löw S et al. Treatment of Ulnar Impaction Syndrome with and without Central TFC Lesion. Wrist Surg 2018; 7: 133 - 140

Das Impingement des triangulären fibrokartilaginären Komplexes (TFCC) zwischen distalem Ende der Ulna und den ulnaren Handwurzelknochen kann zu einem ulnokarpalen Impaktionssyndrom führen. Degenerative Veränderungen bis hin zur manifesten Arthrose im ulnokarpalen und gelegentlich sogar im distalen Radioulnargelenk können die Folge sein.

Bei nachweisbarer zentraler Läsion des TFCC gilt das arthroskopische Debridement mit TFCC-Teilresektion als Standardverfahren. Ist eine degenerative Schädigung jedoch bei der radiokarpalen Arthroskopie nicht nachweisbar, diskutieren die Experten über die adäquate Behandlung: Einige Autoren sind der Meinung, dass auch in diesem Fall die zentrale Resektion des TFCC zu einer Beschwerdebesserung führen kann, andere sind davon nicht überzeugt. Eine deutsche Arbeitsgruppe hat nun die beiden Vorgehensweisen miteinander verglichen.

Die Handchirurgen um Steffen Löw haben zwischen Januar 2012 und Juni 2015 32 Patienten mit ulnarseitigen Handgelenkschmerzen bei Ulna-Impaction-Syndrom arthroskopisch operiert. Bei der klinischen Untersuchung des Gelenks war lediglich ein positives Fovea-Zeichen nachweisbar, alle anderen Befunde waren unauffällig. In allen Fällen erfolgten bei der Arthroskopie ein Debridement und eine Resektion des zentralen Anteils des Handgelenkdiskus, unabhängig von dessen makroskopischem Erscheinungsbild.

Für die jetzige Auswertung beurteilten die Wissenschaftler die postinterventionellen Ergebnisse in Monat 3, 6 und 12 bzw. nach eventuell erforderlichen zu- sätzlichen Eingriffen. Dabei verglichen sie Patienten mit und solche ohne sichtbare TFCC-Schädigung bei der ursprünglichen Arthroskopie. Als Parameter verwendeten sie

- die Funktionsfähigkeit anhand des DASH-Werts (Disabilities of the Arm, Shoulder, and Hand),

- die Schmerzen in Ruhe und bei Belastung auf einer visuellen Analogskala,

- den Bewegungsumfang im Handgelenk sowie bei Pronation/Supination und

- die grobe Kraft.

Darüber hinaus prüften sie, ob im Verlauf weitere operative Eingriffe notwendig geworden waren.

- Bei 16 Patienten hatte laut arthroskopischem Befund eine zentrale TFCLäsion vorgelegen (Gruppe 1),

- bei weiteren 16 Patienten war das nicht der Fall gewesen (Gruppe 2).

Im Hinblick auf Alter, Dauer der Symptomatik und Bestehen einer Arbeitsunfähigkeit waren die beiden Gruppen vergleichbar. Nach einer Nachbeobachtungszeit von im Mittel 1,7 Jahren fanden sich sowohl in Gruppe 1 als auch in Gruppe 2 deutliche Verbesserungen von Schmerzen und Funktionsfähigkeit laut DASH. Dabei hatte der DASH-Punktwert bis Monat 12 und bis zur letzten Nachuntersuchung in Gruppe 2 signifikant stärker abgenommen (entsprechend einer ausgeprägteren Funktionsbesserung), um 36 Punkte gegenüber 23 Punkten in Gruppe 1. Der endgültige Wert lag bei 8,8 Punkten in Gruppe 1 und bei 7,5 Punkten in Gruppe 2. Alle anderen Ergebnisparameter waren zwischen den beiden Gruppen vergleichbar.

Bei 5 der 16 Patienten ohne zentrale TFCLäsion wurde wegen persistierender oder rezidivierter Handgelenkschmerzen ein erneuter Eingriff notwendig. Bei $4 \mathrm{~Pa}-$ tienten erfolgte eine Ulnaverkürzung, bei 1 Patienten eine zweite Arthroskopie mit Debridement und erneuter Resektion des regenerierten Diskus. In der Gruppe der Patienten mit zentraler Diskusläsion wurde in 4 Fällen eine sekundäre Ulnaverkürzungsosteotomie durchgeführt.
Die Patientenzufriedenheit nach dem Eingriff war hoch: In Gruppe 1 waren 13 der 15 auswertbaren Patienten zufrieden, in Gruppe 2 waren es 15 von 16.

FAZIT

Bei etwa drei Viertel der Patienten mit ulnarseitigen Handgelenkschmerzen aufgrund eines Ulna-Impaction-Syndroms führt die arthroskopische Resektion des TFC zu einer Besserung von Schmerzen und Funktion, fassen die Autoren zusammen. Und dieses Ergebnis ist unabhängig davon, ob in der Arthroskopie tatsächlich eine TFC-Läsion sichtbar ist. Bei weiter bestehenden Beschwerden kann die Ulnaverkürzungsosteotomie als Second-Line-Therapie eingesetzt werden.

Dr. Elke Ruchalla, Bad Dürrheim 